\title{
Spacecraft Attitude Control Using Control Moment Gyro Reconfiguration
}

\author{
Kanthalakshmi SRINIVASAN ${ }^{1}$, Deepana GANDHI ${ }^{1}$, Manikandan VENUGOPAL ${ }^{2}$ \\ ${ }^{1}$ Department of Instrumentation and Control Systems Engineering, \\ PSG College of Technology, \\ Coimbatore - 641004, India, \\ klakshmiramesh@yahoo.co.in,deepana.gandhi@gmail.com \\ 2 Department of Electrical and Electronics Engineering, \\ Coimbatore Institute of Technology, \\ Coimbatore - 641014 , India, \\ manikantan-cit@gmail.com
}

\begin{abstract}
The orientation of a satellite in space described relative to some other object or system is known as the attitude of the satellite. The attitude may be changing with time. To be able to control the attitude of the satellite, it must be equipped with actuators that can produce the required torque. Control Moment Gyroscope is a space craft control actuator which acts as torque amplifier. It is suitable for three axis slew manoeuvring by providing the necessary torques via gimbaling a spinning flywheel. Control Moment Gyroscope is considered to be more efficient in terms of power consumption and slew rate. A major drawback encountered with the use of the Control Moment Gyroscope is the possibility of singularities for certain combinations of gimbal angles. The objective of this work is to detect these singularities so that robust steering laws can be developed.
\end{abstract}

Keywords: Control Moment Gyroscope, Gimbal angles, Singularities, Attitude Control, Steering laws.

\section{Introduction}

With the development of space technologies, different classes of satellites are constructed and utilized for various space missions. From the moment that a satellite is launched into its initial orbit, commonly called a transfer orbit, multiple attitude and orbital changes must be performed to obtain the desired final orbit. The motion of a spacecraft can be divided into two types: translational motion and rotational motion. Translational motion describes the motion of a spacecraft in an orbit, whereas the rotational motion describes the orientation (attitude) of the spacecraft in space with respect to a particular reference like the earth and sun. The Attitude control is the process of orienting the spacecraft in a specified predetermined direction. It is an important subsystem in a spacecraft and is responsible for pointing and slewing of the spacecraft. The spacecraft is considered to be a rigid body whose attitude can be described by Euler's equations of rotational motion which govern the rotational dynamics of rigid bodies.

Their solution gives the angular velocity vector due to applied torque and kinematic differential equations, which relate the time derivatives of the orientation angles to the angular velocity vector. Attitude representation can be done using three or four parameters. The threeparameter attitude representation techniques are Euler angles, Gibbs vector, Cayley Rodrigues vector and Modified Rodrigues parameter. The four-parameter representation of attitude is given by the unit quaternion (Euler parameters).

Attitude is the three-dimensional orientation of a vehicle with respect to a specified reference frame. Attitude systems include the sensors, actuators, avionics, algorithms, software, and ground support equipment used to determine and control the attitude of a vehicle. Spacecraft attitude changes according to the fundamental equations of motion for rotational dynamics, the Euler equations.

Attitude determination is the process of combining available sensor inputs with knowledge of the spacecraft dynamics to provide an accurate and unique solution for the attitude state as a function of time, either onboard for immediate use, or after the fact (i.e. post-processing).

Attitude control is the combination of the prediction of and reaction to a vehicle's rotational dynamics. Because spacecraft exist in an environment of small and often highly predictable disturbances, they may in certain cases be passively controlled. Alternately, a spacecraft may include actuators that can be 
used to actively control orientation. These two general types of attitude control are not mutually exclusive.

A general framework for the analysis of the attitude tracking control problem for a rigid body is presented in [1]. A survey of attitude representations in a single consistent notation and set of conventions is given in [2]. The relations between the various representations of the attitude and the kinematic equations are given completely. The transformations connecting different attitude covariance representations are presented by an infinitesimal rotation.

A case study on spacecraft attitude control is presented in [3]. The plant (spacecraft attitude model) is a second order, nonlinear, multiinput-multi-output system defined by Euler's equations of rotational motion and the kinematic differential equations. The Modified Rodrigues parameter (MRP) is used for kinematic parameterization and is the only measurable variable at the plant output.

Control Moment Gyroscopes (CMGs) are proposed in [4] as torque actuators that can effectively answer the attitude control requirements of future spacecraft. The CMG's technology presented can greatly improve the torque and momentum capability of spacecraft's and consequently their agility.

The work on CMG based AOCS is of great interest for past few years. A top-down analysis strategy so as to define the right product future missions, starting from review of system needs in Earth observation and scientific missions to the preliminary mechanism design is presented in [5]. An innovative solutions, which simplify the system, the operations and the CMG mechanisms is presented. A cluster of smallsized single-gimbal control moment gyros (SGCMGs) is proposed in [8] as an attitude control actuator for high-speed manoeuvre of small satellites.

Two objectives dominate consideration of control moment gyroscopes for spacecraft manoeuvres: high torque (equivalently momentum) and singularity-free operations. A significant body of research toward these two goals using a minimal three-control-momentgyroscope array to provide significant singularity-free momentum performance increase spherically (in all directions) by modification of control-moment-gyroscope skew angles is presented in [9], and compared with the ubiquitous pyramid geometry skewed at $54.73 \mathrm{deg}$.

The performance of a control moment gyroscope cluster for 3-axis attitude control for small satellites is presented in paper [10].Experimental results on the performance of a Control Moment Gyroscope (CMG) cluster are presented in [12]. The experimental data are compared with simulation (theoretical) results and both are used to verify the principles, benefits and performance specifications of the CMG cluster.

The major problem encountered with the application of $\mathrm{CMG}$ is the occurrence of singularities for certain combination of gimbal angles. This paper describes the techniques used to detect the occurrence of singularity and the robust steering laws that can be used to overcome singularity.

\section{Spacecraft Dynamics and Kinematics}

It is convenient to think of a spacecraft as being a collection of those particles and bodies that lie within a closed surface $\mathrm{S}$ as shown in Figure 1.

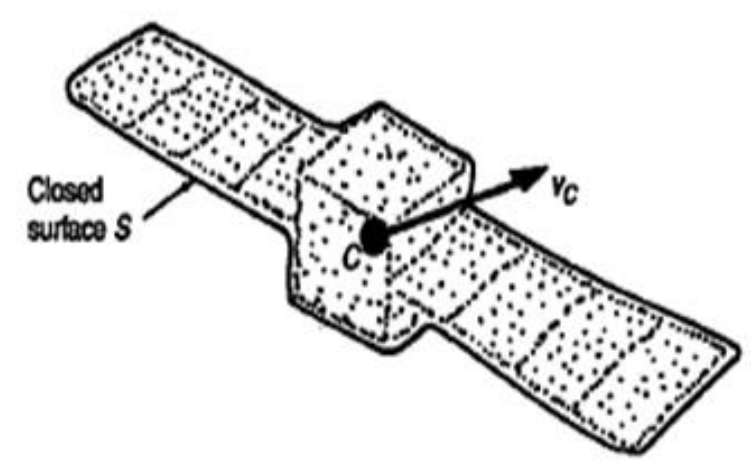

Figure 1. Dynamics of Spacecraft

This concept allows the surface to be chosen at one's convenience, to embrace the complete craft or just part of it or when considering a docking manoeuvre or a tethered configuration. The dynamics of the craft (or whatever is within the surface S) may now be described in terms of its momentum- its linear momentum $\mathrm{L}$ and its angular momentum $\mathrm{H}$ - the former leading to equations that describe the trajectory and the latter leading to equations that describe the attitude motion. In particular, it is the centre-ofmass $\mathrm{C}$ whose trajectory will be described. 


\subsection{The centre-of-mass, $C$}

The centre-of-mass of the particles in S, relative to an arbitrary point 0 , is the point whose position vector $\mathrm{r}_{\mathrm{oc}}$ obeys equation (1).

$\mathrm{Mr}_{\mathrm{oc}}=\sum\left(\mathrm{mr}_{\mathrm{op}}\right)$

where $r_{o p}$ is the position vector of a general particle $P$ shown in Figure 2, $m$ is the mass of the general particle and $M$ is the total mass within $S$.

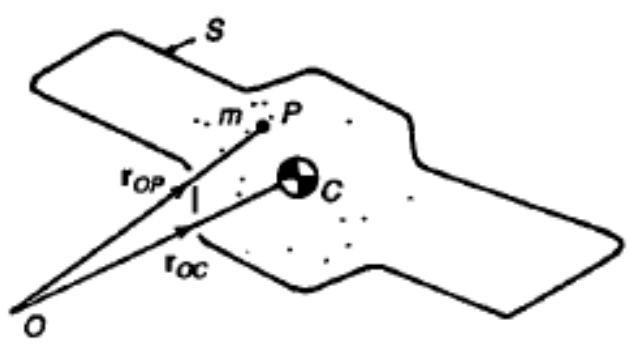

Figure 2. Trajectory Dynamics

When an object has a continuous mass distribution, the integral equivalent of equation (1) should be used, this being

$\mathrm{Mr}_{\mathrm{oc}}=\int \mathrm{r}_{\mathrm{op}} \mathrm{dm}$

In the development of the theory of dynamics, the Centre-of-mass $\mathrm{C}$ is useful as an origin or reference point for the particles in $S$. Putting the origin there leads to $r_{o c}=0$ and so equations (1) and (2) become

$\sum\left(m r_{c p}\right)=0$ and $\int r_{c p} d m=0$

Since this is true at all times, its derivatives are also zero, leading to

$\sum\left(m V_{C P}\right)=0$ and $\int V_{C P} d m=0$

where $V_{C P}$ is the velocity of a particle at $P$ relative to the Centre-of-mass $\mathrm{C}$.

\subsection{General Attitude Dynamics}

Trajectory dynamics supplies rules governing the motion of the Centre-of-mass $\mathrm{C}$ relative to some inertially fixed frame of reference. Attitude dynamics on the other hand uses the Centre-of-mass $\mathrm{C}$ as a reference point.

In terms of momentum, attitude dynamics is mathematically identical to trajectory dynamics. That is to say angular momentum $\mathrm{H}$ responds to a torque $\mathrm{T}$. But the physical motions associated with the two types of momentum are quite different. In order to establish the fundamental principles in terms of momentum, it is convenient to refer once more to the closed surface $S$ (Figure 1), the boundary that separates particles that are of interest from those that are not.

\subsection{Angular momentum (H)}

The angular momentum $H o$ referred to a point 0 is defined as the aggregate of the moments of the momenta of all the particles within S. In mathematical terms, angular momentum referred to 0 is defined as

$\mathrm{H}_{\mathrm{o}}=\sum(\mathrm{r} \times \mathrm{mv})$

where both $\mathrm{r}$ and $\mathrm{v}$ are relative to $\mathrm{O}$. In physical terms the angular momentum of a rigid body is a measure of the torque impulse that is needed to create its rotational motion. Thus the rotation of a body will be brought to rest by the application of a torque impulse that is equal and opposite to its angular momentum $\mathrm{H}$. The reference point that is most useful for attitude dynamics is the Centre-of-mass $\mathrm{C}$. The rule governing the transfer of reference point from $\mathrm{C}$ to some other point 0 is

$\mathrm{H}_{\mathrm{o}}=\mathrm{H}_{\mathrm{c}}+\mathrm{Mh}_{\mathrm{o}}=\mathrm{H}_{\mathrm{c}}+(\mathrm{r} \times \mathrm{Mr})$

Figure 3 illustrates the terms used. The transfer involves adding on the moment-of-momentum to the transfer of inertia from $\mathrm{C}$ to 0 . Equation (6) could be used, for example, for expressing the contribution to a spacecraft's total angular momentum referred to its Centre-of-mass, which arises from a momentum wheel.

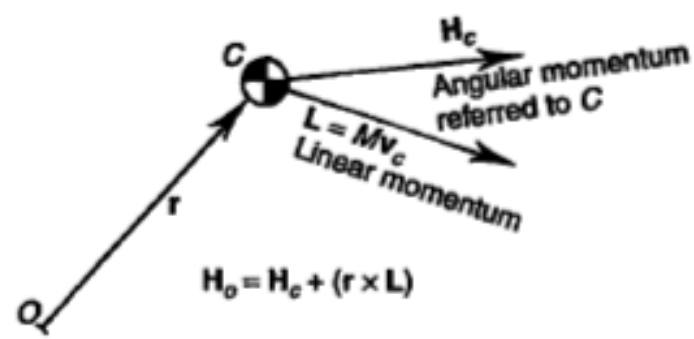

Figure 3. Transfer of reference point from $\mathrm{C}$ to $\mathrm{O}$.

\subsection{Rate of change of angular momentum}

The following explanation applies only when the reference point is the Centre-of-mass $\mathrm{C}$ or an inertially fixed points 1 . It does not apply for other points. The angular momentum may be changed in two ways, the first being:

1. By applying an external couple or a force that has a moment about the reference point. Its consequence is a rate of change of $\mathrm{H}$. referred to $\mathrm{C}$ or $I$, described by the Newtonian equation 
$\frac{\mathrm{d}(\mathrm{H})}{\mathrm{dt}}=\mathrm{T}$

Internal torques, acting between particles or bodies, will not change the total momentum. Thus mechanisms, fuel movement and so on will not change the total angular momentum of a spacecraft. Similarly, the forces between two docking spacecraft will not affect their combined angular momentum.

The second means of changing $\mathrm{H}$ is:

2. By the ejection of some particles from $S$ whose momenta have moments about the reference point. This will occur during the firing of rockets, when their thrust vector does not pass precisely through the Centreof-mass.

As the angular momentum $\mathrm{H}$ becomes very large, the effect of a torque impulse becomes less, leading to the property known as gyroscopic rigidity. This characteristic is made use of by spacecraft designers when they give their craft momentum bias, as a means of making the bias direction insensitive to disturbance torques. Making $\mathrm{H}$ large causes the precession rate to become small in response to a given torque. The rate of change of the components of $\mathrm{H}$ depends upon the rotation of the axis system chosen, in addition to the change described by the Newtonian equation (7). When the axis system has an angular velocity 0 relative to inertial space, then equation (7) must be interpreted using the Coriolis theorem, as

$\mathrm{d}\left(\mathrm{H}_{\mathrm{C}}\right) \mathrm{dt}=\frac{\mathrm{d}}{\mathrm{dt}_{\text {compts }}\left(\mathrm{H}_{\mathrm{C}}\right)}+\left(\Omega \times \mathrm{H}_{\mathrm{C}}\right)=\mathrm{T}$

where $\frac{\mathrm{d}}{\mathrm{dt}_{\text {compts }}}$ means the rate of change of the components of $\left(\mathrm{H}_{\mathrm{C}}\right)$.

\subsection{Angular momentum of rigid bodies}

The angular momentum $\mathrm{H}_{\mathrm{C}}$ of a single rigid body referred to its Centre-of-mass $\mathrm{C}$ maybe expressed as

$\mathrm{H}_{\mathrm{C}}=\left[\mathrm{I}_{\mathrm{C}}\right] \omega$

where $\omega$ is its angular velocity relative to an inertial (non-rotating) frame of reference and $\left[\mathrm{I}_{\mathrm{C}}\right]$ is the inertia matrix based upon the centreof-mass C. In general, $\left[I_{C}\right]$ may be expressed as

$\left[I_{C}\right]=\left[\begin{array}{ccc}I_{x x} & -I_{x y} & -I_{z x} \\ -I_{x y} & I_{y y} & -I_{y z} \\ -I_{z x} & -I_{z y} & I_{z z}\end{array}\right]$ where $\mathrm{I}_{\mathrm{xx}}, \mathrm{I}_{\mathrm{yy}}$ and $\mathrm{I}_{\mathrm{zz}}$ are the moments of inertia. $\mathrm{I}_{\mathrm{xy}}, \mathrm{I}_{\mathrm{yz}}$ and $\mathrm{I}_{\mathrm{zx}}$ are the products of inertia, broadly representing a measure of the lack of mass symmetry, leading to crosscoupled behaviour. Everybody has a set of orthogonal axes at each point, for which the products of inertia are zero. These are called principal axes, and there may be more than one set of them, depending upon the mass symmetry of the body. Principal axes are eigenvectors of the inertia matrix.

It is evident from equation (9) that the components of the angular momentum may in general be expressed as

$H_{C}=\left[\begin{array}{ccc}\left(I_{x x} \omega_{x}\right. & -I_{x y} \omega_{y} & \left.-I_{x x} \omega_{z}\right) \\ \left(I_{y y} \omega_{y}\right. & -I_{y z} \omega_{z} & \left.-I_{x y} \omega_{x}\right) \\ \left(I_{z z} \omega_{z}\right. & -I_{z x} \omega_{x} & \left.-I_{y z} \omega_{y}\right)\end{array}\right]$

When principal axes are used, then

$\mathrm{H}_{\mathrm{C}}=\left[\begin{array}{lll}\mathrm{I}_{\mathrm{xx}} \omega_{\mathrm{x}} & \mathrm{I}_{\mathrm{yy}} \omega_{\mathrm{y}} & \mathrm{I}_{\mathrm{zz}} \omega_{\mathrm{z}}\end{array}\right]^{\mathrm{T}}$

\subsection{Rotational kinetic energy}

The rotational energy of a rigid body may be expressed as

$E=\frac{1}{2}\left(H_{C} . \omega\right)$ or $\frac{1}{2}\left(I_{C} \omega . \omega\right)$

work is done by a torque $T$ at a rate $T$. $\omega$, and the rate at which the rotational energy is increased by a torque $T_{C}$ about $C$ is $T_{C} . \omega$. It follows that in the absence of any external torque $T_{e}$ the rotational energy will remain constant.

\subsection{Equations of motion}

The equation of motion of attitude dynamics can be divided into two sets: the kinematics equations of motion and the dynamic equations of motion. Dynamics is the study of motion irrespective of the forces that bring about the motion. The kinematics equations of motion are a set of first-order differential equations specifying the time evolution of the attitude parameters.

\subsection{Dynamic equations of motion}

The basic equation of attitude dynamics relates the time derivative of the angular momentum vector $\mathrm{dH}$, to the applied torque $\mathrm{N}$.

The rate of change angular momentum

$\frac{\mathrm{dH}}{\mathrm{dt}}=\mathrm{N}-\omega * \mathrm{H}=\mathrm{I} \frac{\mathrm{d} \omega}{\mathrm{dt}}$

where the torque vector, $N$, is defined as: 


$$
\mathrm{N} \equiv \sum_{\mathrm{i}=1}^{\mathrm{n}} \mathrm{r}_{\mathrm{i}} \mathrm{xF}_{\mathrm{i}}
$$

and $\omega$ is instantaneous angular velocity vector. The force $F_{i}$, on the ith mass consists of two parts: an externally applied force $\mathrm{F}_{\mathrm{i}}^{\text {ext }}$, and internal force consisting of the number of forces $F_{i j}$, exerted by the other masses

$F_{i}=F_{i}^{e x t}+\sum_{j=1 ; j \neq i}^{n} F_{i j}$

Thus

$\mathrm{N}=\sum_{\mathrm{i}=1}^{\mathrm{n}} \mathrm{r}_{\mathrm{i}} \times \mathrm{F}_{\mathrm{i}}^{\mathrm{e} \times \mathrm{t}}+\sum_{\mathrm{i}=1}^{\mathrm{n}} \sum_{\mathrm{j}=1 ; \mathrm{j} \neq \mathrm{i}}^{\mathrm{n}} \mathrm{r}_{\mathrm{i}} \times \mathrm{F}_{\mathrm{ij}}$

Each pair of masses contributes two terms to the second number, $r_{i} \times F_{i j}$ and $r_{j} \times F_{j i}$.By newton's third law of motion $F_{j i}=-F_{i j}$, so the contribution to the sum of each pair of masses is $\left(r_{i}-r_{j}\right) \times F_{i j}$. If the line of action of the force between each pair of masses is parallel to the vector between the masses, $\left(r_{i}-r_{j}\right)$, the cross product vanishes, and the net torque $\mathrm{N}$, is equal to the torque due to external forces alone. This is always assumed to be the case in spacecraft applications.

Equation (14) is the fundamental equation of rigid body dynamics. The presence of the $\omega \times$ $H$ term on the right side means that $\mathrm{H}$, and hence $\omega$, is not constant in the spacecraft frame, even if $\mathrm{N}=0$ and the resulting motion is called nutation. Rotational motion without nutation occurs only if $\omega$ and $\mathrm{H}$ are parallel, that is, only if the rotation is about a principal axis of rigid body.

Using $\mathrm{H}=\mathrm{I} \omega$ in equation (14)

$\mathrm{I} \frac{\mathrm{d} \omega}{\mathrm{dt}}=\mathrm{N}-\omega \times(\mathrm{I} \omega)$

or

$\frac{\mathrm{dH}}{\mathrm{dt}}=\mathrm{N}-\left(\mathrm{I}^{-1} \times \mathrm{H}\right) \times \mathrm{H}$

In the principal axis system, equation (18) has the components:

$$
\begin{aligned}
& I_{1}\left(\frac{d \omega_{1}}{d t}\right)=N_{1}+\left(I_{2}-I_{3}\right) \omega_{2} \omega_{3} \\
& I_{2}\left(\frac{d \omega_{2}}{d t}\right)=N_{2}+\left(I_{3}-I_{1}\right) \omega_{3} \omega_{1} \\
& I_{3}\left(\frac{d \omega_{3}}{d t}\right)=N_{3}+\left(I_{1}-I_{3}\right) \omega_{1} \omega_{2}
\end{aligned}
$$

and above equation can be written as:

$$
\begin{aligned}
& \frac{\mathrm{dH}_{1}}{\mathrm{dt}}=\mathrm{N}_{1}+\left(\frac{1}{\mathrm{I}_{2}}-\frac{1}{\mathrm{I}_{3}}\right) \mathrm{L}_{2} \mathrm{~L}_{3} \\
& \frac{\mathrm{dH}_{2}}{\mathrm{dt}}=\mathrm{N}_{2}+\left(\frac{1}{\mathrm{I}_{3}}-\frac{1}{\mathrm{I}_{1}}\right) \mathrm{L}_{3} \mathrm{~L}_{1} \\
& \frac{\mathrm{dH}_{3}}{\mathrm{dt}}=\mathrm{N}_{3}+\left(\frac{1}{\mathrm{I}_{1}}-\frac{1}{\mathrm{I}_{2}}\right) \mathrm{L}_{1} \mathrm{~L}_{2}
\end{aligned}
$$

The dynamic equation of motion of rigid $\mathrm{s} / \mathrm{c}$ are given by Euler's equation as

$\frac{\mathrm{dH}}{\mathrm{dt}}=\mathrm{N}_{\text {Dist }}+\mathrm{N}_{\text {Ctrl }}-\omega \times \mathrm{H}$

where I is the moment of inertia tensor and $\omega$ is the spacecraft angular velocity vector. The time derivative is taken and the vectors are resolved in a body - fixed coordinate system. The terms $\mathrm{N}_{\text {Dist }}+\mathrm{N}_{\text {ctrl }}$ are disturbance and control torques, respectively, acting on the spacecraft.

\subsection{Kinematic equations of motion}

The kinematic equation be written in differential form using the quaternion representation of the attitude as:

$\frac{\mathrm{dq}}{\mathrm{dt}}=\frac{1}{2} \Omega \mathrm{q}$

where $\Omega=\left[\begin{array}{cccc}0 & \omega_{3} & -\omega_{2} & \omega_{1} \\ -\omega_{3} & 0 & \omega_{1} & \omega_{2} \\ \omega_{2} & -\omega_{1} & 0 & \omega_{3} \\ -\omega_{1} & -\omega_{2} & -\omega_{3} & 0\end{array}\right]$

The quaternion representation is generally preferred to the Euler angles representation because of its analytical characteristics.

As the dynamic complexity of spacecraft increases, each degree of freedom must be represented by its appropriate equation of motion. For example incorporating momentum or reaction wheels for attitude stability and manoeuvring adds additional degree of freedom. Momentum wheel dynamics can be included as an additional term in Euler's equation and an additional equation of motion for the wheels themselves. Equation (22) is rewritten as:

$\frac{\mathrm{d}}{\mathrm{dt}}(\mathrm{I} \omega)=\mathrm{N}_{\text {Dist }}+\mathrm{N}_{\text {Crrl }}-\omega \times \mathrm{I} \omega-\left[\omega \times \mathrm{h}+\mathrm{N}_{\text {WHEEL }}\right]$

where $\mathrm{h}$ is the total angular momentum of the reaction wheels and $N_{W H E E L}$ is the net torque applied to the momentum wheels, which is a function of bearing friction, wheel speed and applied wheel motor voltage. The equation of motion of the wheels is 
$\frac{\mathrm{dh}}{\mathrm{dt}}=\mathrm{N}_{\text {WHEEL }}$

The dynamic and kinematic equation of motion are taken as a set of coupled differential equation and integrated.

The capability to attitude-manoeuvre a satellite is based on using control torques. The most common control laws include Euler angles based control laws and quaternion error terminologies based control laws. The simplest torque control law is based on Euler angle errors. The major shortcoming of this law is that the six off-diagonal elements of the error matrix must be computed continuously.

The quaternion error vector expresses the attitude error between (i) the satellite attitude direction in space and (ii) the target direction toward which the satellite is oriented at the end of the attitude manoeuvre. The quaternion error vector is written as:

$$
\left[A\left(\mathrm{q}_{\mathrm{E}}\right)\right]=\left[\mathrm{A}\left(\mathrm{q}_{\mathrm{T}}\right)\right]\left[\mathrm{A}\left(\mathrm{q}_{\mathrm{S}}\right)\right]^{-1}=\left[\mathrm{A}\left(\mathrm{q}_{\mathrm{T}}\right)\right]\left[\mathrm{A}\left(\mathrm{q}_{\mathrm{S}}^{-1}\right)\right]
$$

The attitude control laws are given by:

$$
\begin{aligned}
\mathrm{T}_{\mathrm{cx}} & =2 \mathrm{~K}_{\mathrm{x}} \mathrm{q}_{1 \mathrm{E}} \mathrm{q}_{4 \mathrm{E}}+\mathrm{K}_{\mathrm{xd}} \mathrm{p}, \\
\mathrm{T}_{\mathrm{cy}} & =2 \mathrm{~K}_{\mathrm{y}} \mathrm{q}_{2 \mathrm{E}} \mathrm{q}_{4 \mathrm{E}}+\mathrm{K}_{\mathrm{yd}} \mathrm{q}, \\
\mathrm{T}_{\mathrm{cz}} & =2 \mathrm{~K}_{\mathrm{z}} \mathrm{q}_{3 \mathrm{E}} \mathrm{q}_{4 \mathrm{E}}+\mathrm{K}_{\mathrm{zd}} \mathrm{r}
\end{aligned}
$$

As this method requires fewer algebraic operations, it is the most preferred control law.

\section{Control Moment Gyros - Actuator for Spacecraft Attitude Control}

A Control Moment Gyro (CMG) (Figure 4) is a torque generator for attitude control of an artificial satellite in space. Also it is a momentum exchange device which is used as an actuator for spacecraft attitude control. It rivals a reaction wheel in its high output torque and rapid response.

A CMG consists of a flywheel rotating at a constant speed, one or two supporting gimbals, and motors which drive the gimbals. A rotating flywheel possesses angular momentum with a constant vector length. Gimbal motion changes the direction of this vector and thus generates a gyro-effect torque. The working principle of CMG installed on a spacecraft can be described as a rotor that spins at a constant speed and is gimballed to apply a gyroscopic torque on the spacecraft. When the gimbal is rotated, the spin axis of the rotor points along different directions causing a change in its angular momentum orientation. The gyroscopic torque is proportional to the rate of change of the angular momentum.

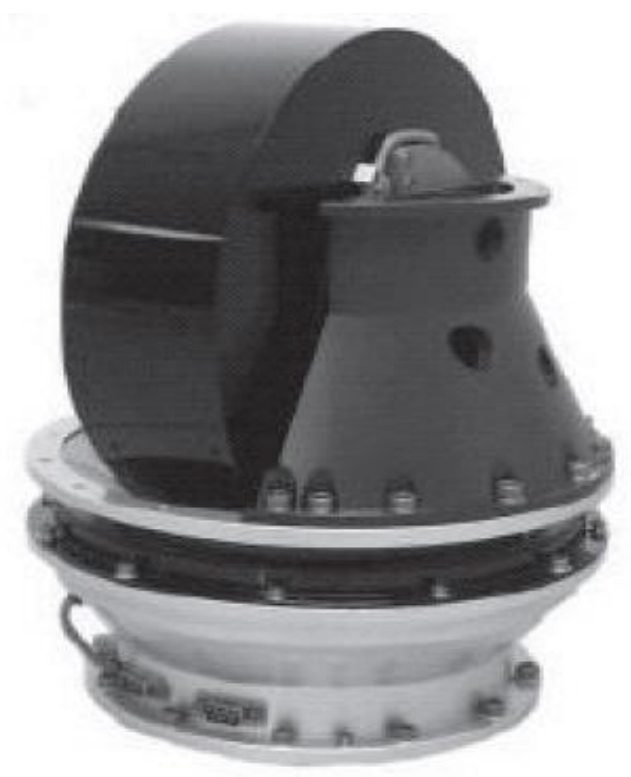

Figure 4. Control Moment Gyro

There are two types of CMG units: single gimbal and double gimbal. A single gimbal CMG generates a one axis torque and a double gimbal CMG generates a two axis torque. In both cases, the direction of the output torque changes in accordance with gimbal motion. For this reason, a system composed of several units is usually required to obtain the desired torque.

\section{Mathematical Formulation}

A generalized system is considered consisting of $n$ identically sized single gimbal CMG units. The number $n$ is not less than 3 to enable three axis control. The system configuration is defined by the relative arrangement of the gimbal directions. The system state is defined by the set of all gimbal angles, each of which are denoted by $\delta_{i}$. Three mutually orthogonal unit vectors are shown in Figure 5a. and are defined as follows:

$g_{i}:$ Gimbal vector, $c_{i}$ :Torque vector

$h_{i}$ : Normalized angular momentum vector, where

$\mathrm{c}_{\mathrm{i}}=\partial \mathrm{h}_{\mathrm{i}} / \partial \delta_{\mathrm{i}}=\mathrm{g}_{\mathrm{i}} \times \mathrm{h}_{\mathrm{i}}$ 


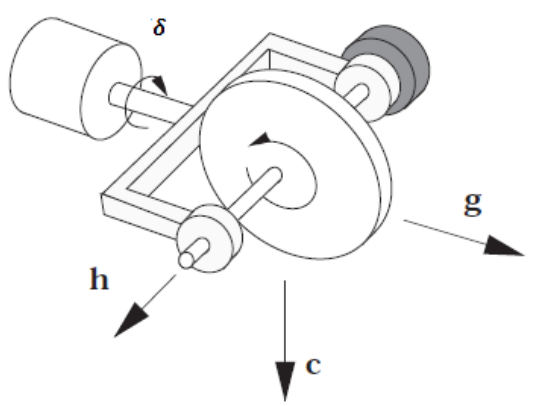

Figure 5a. Ortho normal vectors of a CMG unit

The gimbal vectors are constant while the others are dependent upon the gimbal angle $\delta_{i}$. Once the initial vectors are defined as in Figure $5 \mathrm{~b}$, the other vectors are obtained as follows:

$$
\begin{aligned}
& \mathrm{h}_{\mathrm{i}}=\mathrm{h}_{\mathrm{i} 0} \cos \delta_{\mathrm{i}}+\mathrm{c}_{\mathrm{i} 0} \sin \delta_{\mathrm{i}}, \\
& \mathrm{c}_{\mathrm{i}}=-\mathrm{h}_{\mathrm{i} 0} \sin \delta_{\mathrm{i}}+\mathrm{c}_{\mathrm{i} 0} \cos \delta_{\mathrm{i}}
\end{aligned}
$$

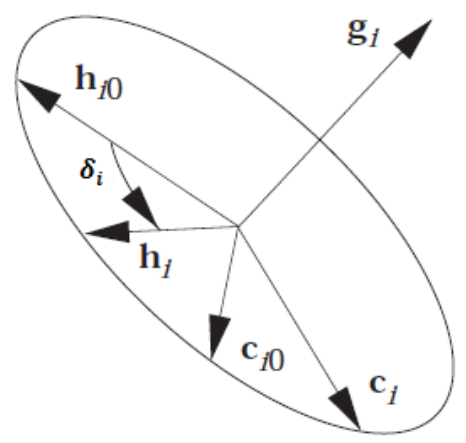

Figure 5b. Gimbal angle and vectors

The total angular momentum is the sum of all $h_{i}$ multiplied by the unit's angular momentum value which is denoted by $h$. In this work, $\mathrm{H}$ denotes the total angular momentum without the multiplier $h$ :

$\mathrm{H}=\sum \mathrm{h}_{\mathrm{i}}$

This relation is simply written as a nonlinear mapping from the set of $\delta_{i}$ to $\mathrm{H}$

$\mathrm{H}=\mathrm{f}(\boldsymbol{\delta})$

The variable, $\delta=\left(\delta_{1}, \delta_{2}, \ldots, \delta_{\mathrm{n}}\right)$, is a point on an $n$ dimensional torus denoted by $T(n)$ which is the domain of this mapping. The mapping range is a subspace of the physical Euclidean space and is denoted by $H$. This space is the maximum workspace. By the analogy of this relation with a spatial link mechanism, this relation will be called kinematics or kinematic equation.

The output torque without the multiplier $h$ is obtained by taking the time derivative as follows:

$$
\mathrm{T}=-\mathrm{dH} / \mathrm{dt}=-\sum \frac{\partial \mathrm{h}_{\mathrm{i}}}{\partial \delta_{\mathrm{i}}} \times \frac{\mathrm{d} \delta_{\mathrm{i}}}{\mathrm{dt}}
$$

The total output torque is a sum of output of each unit, it is also given as:

$\mathrm{T}=-\sum \mathrm{c}_{\mathrm{i}} \omega_{\mathrm{i}}=-\mathrm{C} \omega$

where

$\omega_{\mathrm{i}}=\frac{\mathrm{d} \delta_{\mathrm{i}}}{\mathrm{dt}}$, and $\omega=\left(\omega_{1}, \omega_{2}, \ldots, \omega_{\mathrm{n}}\right)^{\mathrm{t}}$

The variable $\omega_{i}$ is the rotational rate of each gimbal. The vector $\mathrm{w}$ is a component vector of a tangent space of $T(n)$.

\subsection{Steering Law}

The 'steering law' functions to compute the gimbal rates, $\omega$ necessary to produce the desired torque, $\mathrm{T}_{\text {com }}$, and is generally given as a solution of the linear equation given in Equation (32).

$$
\omega=-C^{t}\left(\mathrm{CC}^{\mathrm{t}}\right)^{-1} \mathrm{~T}_{\text {com }}+\left(\mathrm{I}-\mathrm{C}^{\mathrm{t}}\left(\mathrm{CC}^{\mathrm{t}}\right)^{-1} \mathrm{C}\right) \mathrm{k}
$$

where $\mathrm{I}$ is the $\times n$ identity matrix and $\mathrm{k}$ is an arbitrary vector of $n$ elements.

The first term has the minimum norm among all solutions to the equation. The matrix $\mathrm{C}^{\mathrm{t}}\left(\mathrm{CC}^{\mathrm{t}}\right)^{-1}$ is called a pseudo-inverse matrix. The second term, denoted by $\omega_{N}$, is a solution of the homogeneous equation:

$$
\mathrm{C} \omega_{\mathrm{N}}=0
$$

This implies that the motion by this $\omega_{N}$ does not generate a torque $(\mathrm{T})$ and keeps the angular momentum $(\mathrm{H})$ constant. In this sense, this term is called a 'null motion'. The null motion has $n-3$ degrees of freedom because it is an element of the kernel of the linear transformation represented by $C$.

\subsection{Singularity}

A singularity is encountered when there exists some direction in the body in which the array of CMGs is not capable of producing torque. This phenomenon occurs when the gimbal angles of CMGs become some specific arrangement.

It is assumed each CMG has equal and constant angular momentum of unit magnitude. Total output torque for this system is given by the time rate of change of total angular momentum vector:

$\tau=\mathrm{h}=\mathrm{A}(\delta) \dot{\delta}$ 
where A instantaneous Jacobian matrix is:

$$
A(\delta)=\frac{\partial h}{\partial \delta}=\left[\begin{array}{cccc}
-\cos \beta \cos \delta_{1} & \sin \delta_{2} & \cos \beta \cos \delta_{3} & -\sin \delta_{4} \\
-\sin \delta_{1} & -\cos \beta \cos \delta_{2} & \sin \delta_{3} & \cos \beta \cos \delta_{4} \\
\sin \beta \cos \delta_{1} & \sin \beta \cos \delta_{2} & \sin \beta \cos \delta_{3} & \sin \beta \cos \delta_{4}
\end{array}\right]
$$

Each column of the Jacobian matrix represents the output torque vector of the respective CMG in the cluster. The $3 \times$ Jacobian matrix $A$ is a function of the gimbal angle, as shown in equation (36), and it has maximum rank of 3. When $\operatorname{rank}(A)=2$, equation (36) deteriorates, where all $a_{i}$ become coplanar and there exists a unit vector $u$ orthogonal to that coplanar plane; i.e.:

$\mathrm{a}_{\mathrm{i}} \cdot \mathrm{u}=0,(\mathrm{i}=1,2, \ldots, \mathrm{n})$

Therefore, the output vector $T$, that is a linear combination of $a_{i}$, is also normal to $u$ and the CMG system cannot produce any momentum along the direction of $u$. There exists a vector $u$ normal to all $a_{i}$ at the singular point. Therefore we select $u$ as parameter and solve this equation with respect to $a_{i}$. Since $a_{i}$ is perpendicular to both $u$ and gimbal-axis vector $g_{i}$,

$\mathrm{a}_{\mathrm{i}}^{\mathrm{S}}=\left\{\begin{array}{cc}\varepsilon_{\mathrm{i}}\left(\mathrm{g}_{\mathrm{i}} \times \mathrm{u}\right) /\left|\mathrm{g}_{\mathrm{i}} \times \mathrm{u}\right| & \left(\mathrm{g}_{\mathrm{i}} \neq \mathrm{u}\right) \\ \text { arbitrary } & \left(\mathrm{g}_{\mathrm{i}}=\mathrm{u}\right)\end{array}\right.$

where, $\varepsilon_{\mathrm{i}}= \pm 1$ and subscript $S$ denotes singular point. Let $u$ be a unit vector of the punctured unit sphere defined as:

$S=\{u:|u|=1\}$

The angular momentum at a singular point is given by the following equation:

$\mathrm{h}_{\mathrm{i}}^{\mathrm{S}}=\mathrm{a}_{\mathrm{i}}^{\mathrm{S}} \times \mathrm{g}_{\mathrm{i}}$

At the singular point, all $h_{i}$ is in the direction that is along with $u$ or $-u$ as close as possible.

\section{Results and Discussion}

In this work a satellite with moments of inertia $\mathrm{I}_{\mathrm{x}}=27, \mathrm{I}_{\mathrm{y}}=17, \mathrm{I}_{\mathrm{z}}=25 \mathrm{~kg}-\mathrm{m}^{2}$ is considered. Satellite dynamic equations used are Euler's momentum equations. The satellite kinematic equations used are the time derivatives of the quaternion vector. The control torques are generated using Quaternion error vector command law.
In many cases the satellite is required to carry out small manoeuvres in order to orient it in a specified direction. Such a case of small attitude command is considered. The attitude commands in the three axis considered are $\left[-6^{\circ},-4^{\circ}, 4^{\circ}\right]$. The open loop and closed loop results are presented in Figure 6 and Figure 7 respectively.

\subsection{Open loop response}
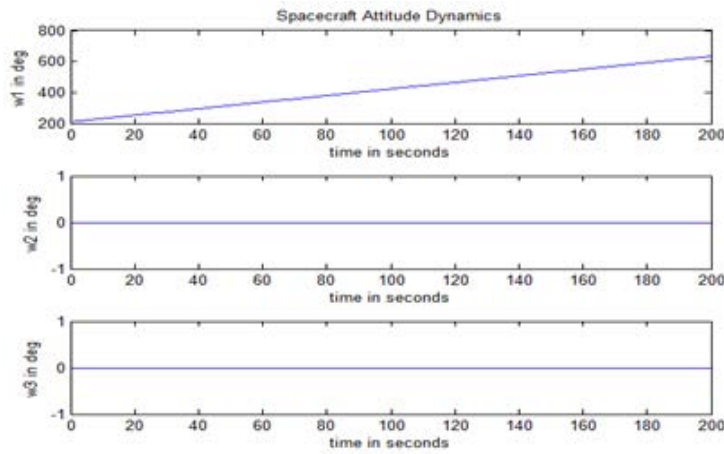

Figure 6a. Angular velocities of the satellite
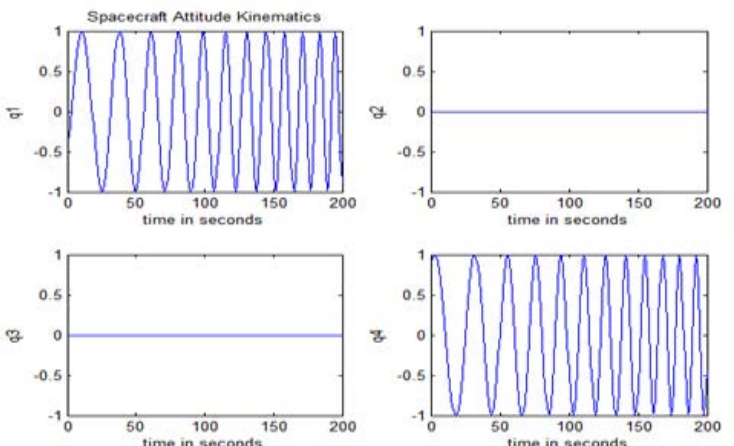

Figure 6b. Quaternion outputs of the satellite
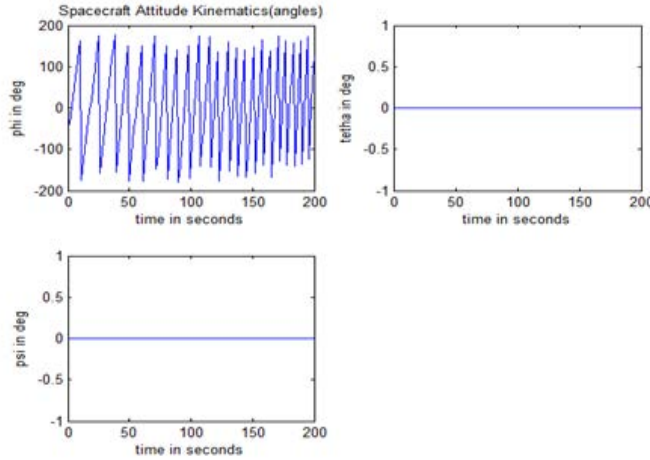

Figure 6c. Time response satellites kinematics 


\subsection{Closed loop response}

Figure $7 \mathrm{a}$ to Figure $7 \mathrm{c}$ that shows the closed loop response reveals that the angular velocities, the quaternion response and the attitude in Euler's angles are stabilised. The control torques and the quaternion errors are shown in Figure $7 \mathrm{~d}$ and Figure $7 \mathrm{e}$. It can be seen that maximum of $0.1 \mathrm{Nm}$ is sufficient for the manoeuvre. The relationship between four quaternions is that sum of squares of quaternions is always one.
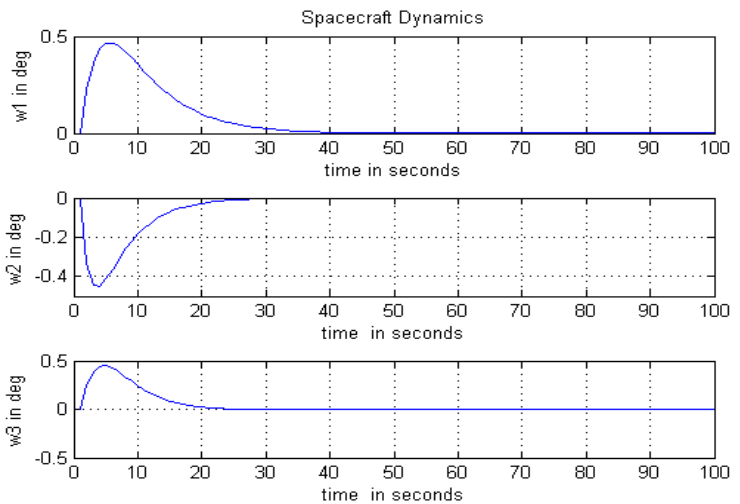

Figure 7a. Angular velocities of the satellite
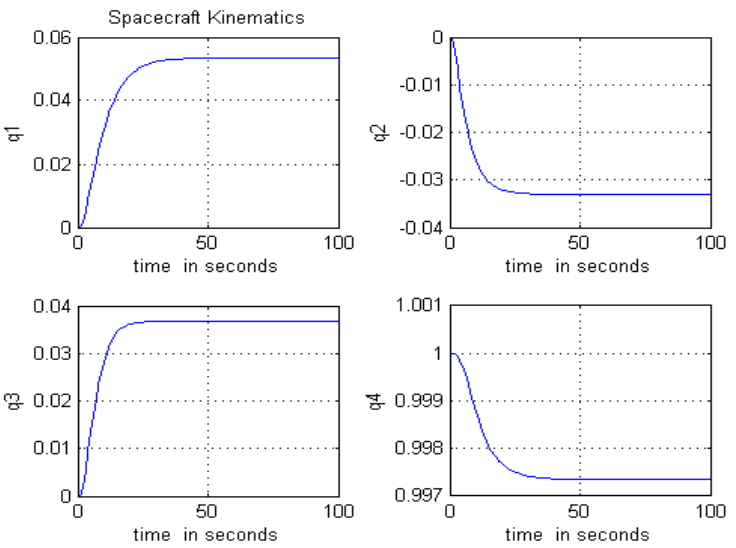

Figure 7b. Quaternion outputs of the satellite
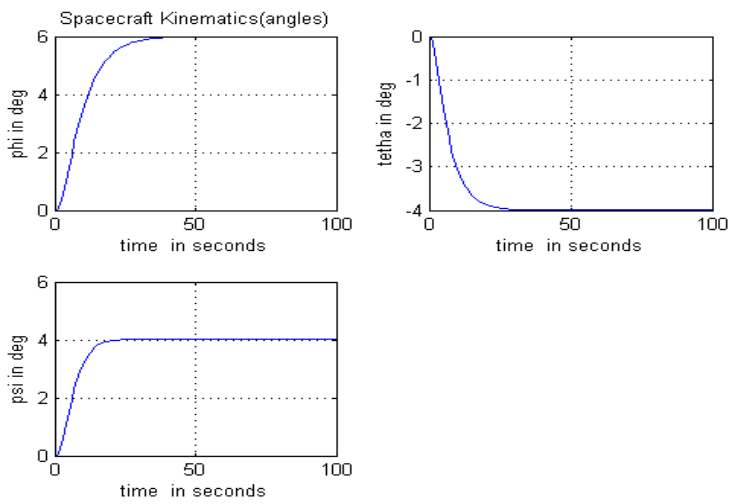

Figure 7c. Time response satellites kinematics
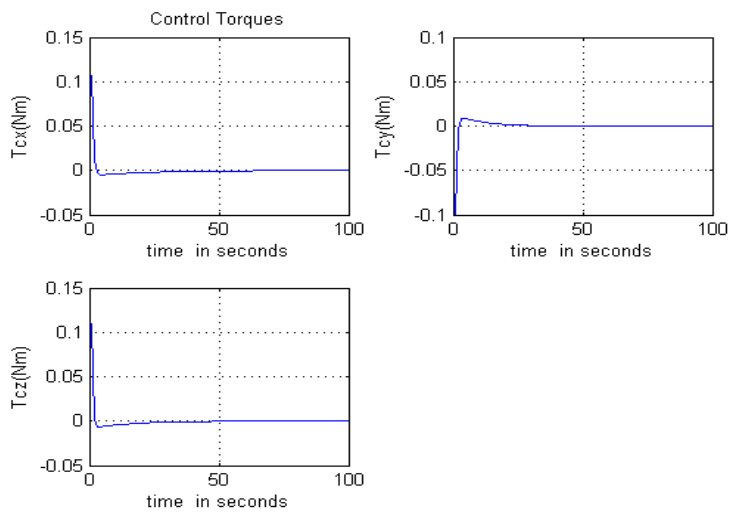

Figure 7d. Control Torques
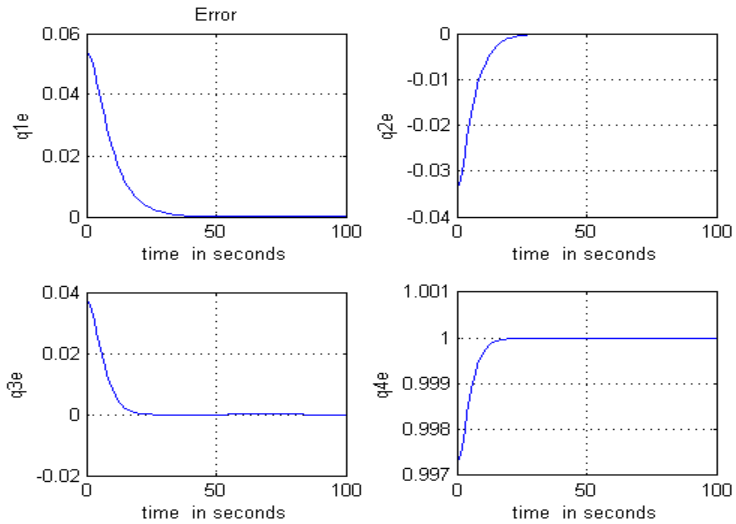

Figure 7e. Quaternion error

\subsection{Singularity detection - effects of singularity on developed torques}

The effects of singularity on developed torques on increasing and sinusoidal control torques are shown below. It can be seen that when singularity occurs the torque values are reducing to zero

\subsubsection{Increasing Torque}

It can be seen from Figure 8. that the actual torque is increasing throughout with respect to time but the developed torque is decreased to zero at the instant when singularity occurs $(\mathrm{t}=20$ seconds).
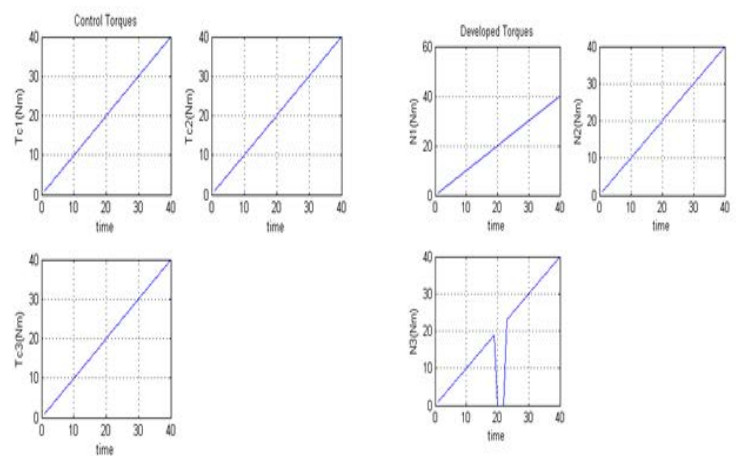

Figure 8. Control Torques and Developed Torques 


\subsubsection{Sinusoidal Torques}

It can be seen from Figure 9. that the actual torque is sinusoidal throughout with respect to time but the developed torque is decreased to zero at the instant when singularity occurs $(t=20$ seconds).
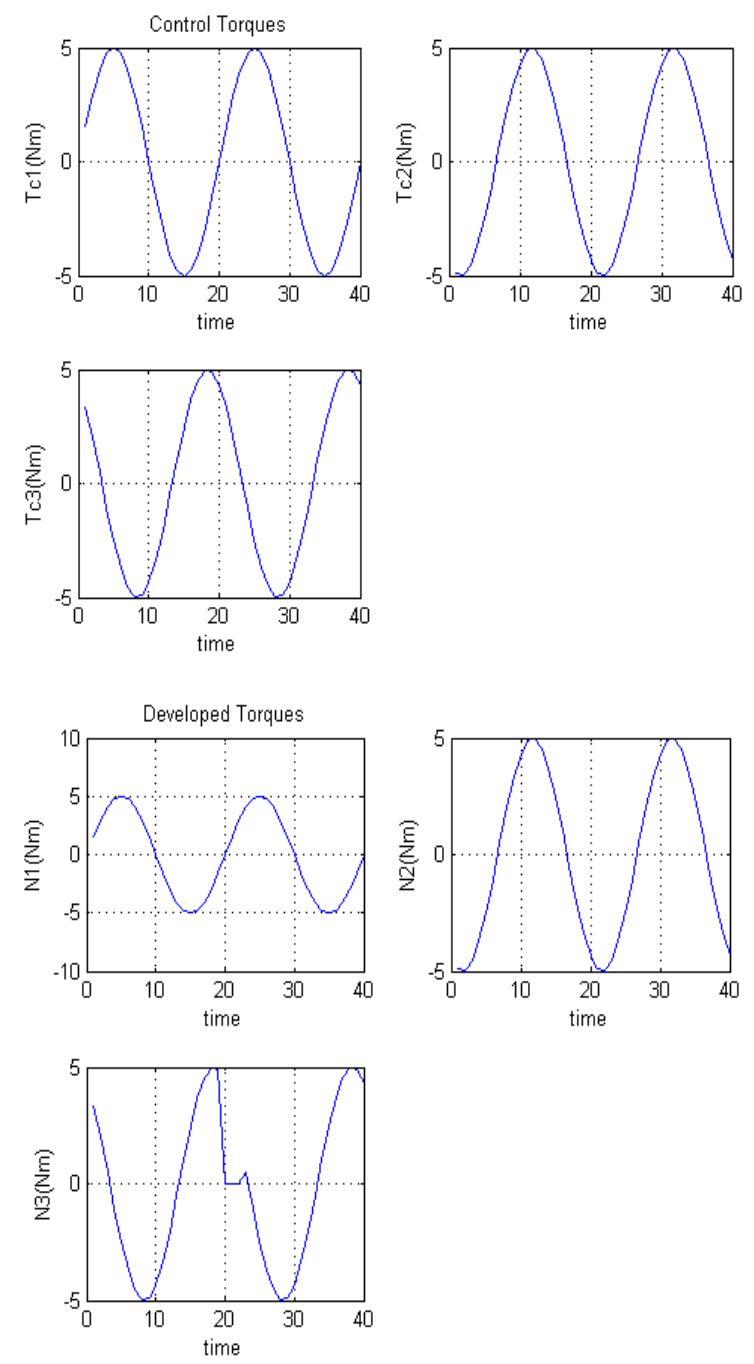

Figure 9. Control Torques and Developed Torques

\subsection{Effect of Singularity on angular velocities}

The changes in angular velocities of satellites due to occurrence of singularity are shown in Figure 10.

From Figure 10, it can be seen that the angular velocity does not change with time when singularity occurs, it saturates for particular interval (time $20-30$ seconds). At that corresponding interval of time the attitude of one axis starts decreasing.
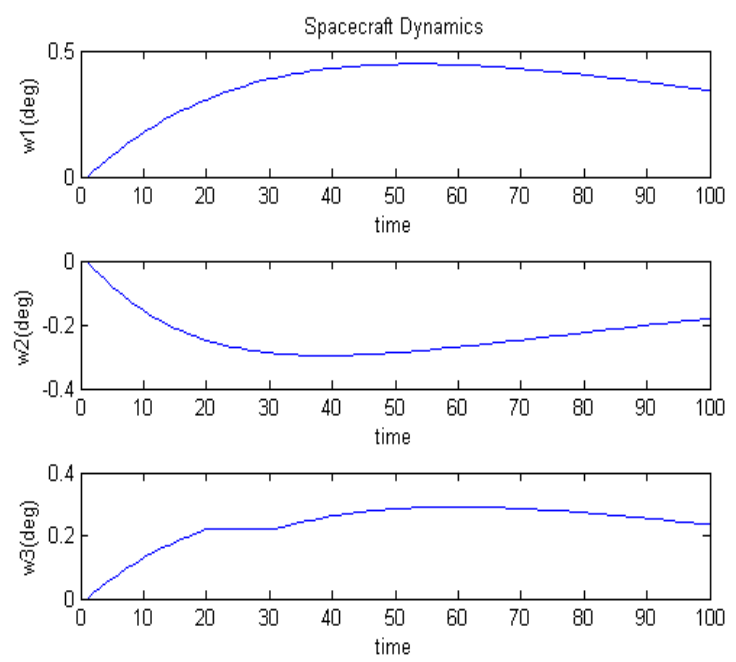

Figure 10. Angular velocities of the satellite

\section{Conclusion}

A control moment gyro (CMG) is a device used as an actuator for attitude control of spacecraft. It generates torques through angular momentum transfer to and from the main spacecraft body. This is achieved by changing the direction of the angular momentum vector of a gimballed flywheel. Because a CMG operates in a continuous manner, it can achieve precise attitude control. Moreover, as it does not consume any propellant, the operational life of the spacecraft can be prolonged.

An obstacle when using a CMG system in practice is the existence of singular gimbal angle states for which the CMGs cannot generate a torque along arbitrary directions. At each singular state, all admissible torque directions lie on a two-dimensional surface in the three-dimensional angular momentum space; therefore, the CMG system cannot generate a torque normal to this surface. These singular states at which a CMG cannot generate torque are detected in this work. Simulation results show that when singularity occurs the developed torque deviates from its original value. In future this work can be extended by providing exact steering laws to avoid singularities so the CMGs can generate a torque even in the presence of singularity. 


\section{REFERENCES}

1. TING-YUNG WEN, J., K. KREUTZDELGADO, The Attitude Control Problem, IEEE Transactions on Automatic Control, vol. 36(10), 1991, pp. 1148-1162.

2. SHUSTER, M. D. A Survey of Attitude Representations, Journal of Astronautical Sciences, vol. 41(4), 1993, pp. 439-517.

3. AHMED, R., D.-W. GU I. POSTLETHWAITE, A Case Study on Spacecraft Attitude Control, IEEE Transactions on Automatic Control, vol.38(9), 2009, pp. 7345-7350.

4. ROSER. X, SGHEDONI. M, Control Moment Gyroscopes (CMG's) and Their Application in Future Scientific Missions, in Proceedings of Third ESA International Conference on Spacecraft Guidance, Navigation and Control Systems, Netherland, 1996, pp. 523-528.

5. DEFENDINI. A., K. LAGADEC, P. GUAY, T. BLAIS, G. GRISERI, Low Cost CMG-based AOCS Designs in the Proceeding of fourth ESA International Conference on Spacecraft Guidance, Navigation and Control Systems, Netherland, 1999, pp. 393-398.

6. WIE, B., D. BAILEY, C. HEIBERG, Singularity Robust Steering Logic for Redundant Single-Gimbal Control Moment Gyros, Journal of Guidance, Control and Dynamics, vol. 24, no. 5, 2001, pp. 865-872.

7. OMAGARI, K., T. USUDA, S. MATUNAGA, Research of Control Moment Gyros for Micro-Satellites and 3-DOF Attitude Dynamics Simulator Experiment, in the Proceedings of the 8th International Symposium on Artificial Intelligence, Robotics and Automation in Space, Munich, 2005, pp. 503-508.

8. OKUBO, H., Y. TANI, Singularity Robust Steering of Redundant Single Gimbal Control Moment Gyros for Small Satellites, in the Proceedings of The 8th International Symposium on Artificial Intelligence, Robotics and Automation in Space, Munich, 2005, pp. 603-609.
9. SANDS, T. A., J. J. KIM, B. N. AGRAWAL, Nonredundant SingleGimbaled Control Moment Gyroscopes, Journal of Guidance, Control and Dynamics, vol. 35(2), 2012, pp. 578-587.

10. AREZKI, M., S. MOHAMMED, Simulation of Three Axis Attitude Control Using a Control Momentum Gyroscope for Small Satellites, in the Proceedings of the World Congress on Engineering, London, 2012, pp. 978-988.

11. FORD, K. A., C. D. HALL, Singular Direction Avoidance Steering for Control-Moment Gyros, Journal of Guidance, Control, and Dynamics, vol. 23, no. 4, 2000, pp. 648-656.

12. LAPPAS, V. J., W. H. STEYN, C. I. UNDERWOOD, Experimental Testing of a CMG Cluster for Agile Microsatellites in the Proceedings of IEEE 11th Mediterranean Conference on Control and Automation, Greece, 2003, pp. 602-610.

13. YOON, H., P. TSIOTRAS, Singularity Analysis of Variable-Speed Control Moment Gyros, Journal of Guidance, Control, and Dynamics, vol. 27, no. 3, 2004, pp. 374-386.

14. YOON, H., P. TSIOTRAS, Spacecraft Line-of-Sight Control Using a Single Variable-Speed Control Moment Gyro, Journal of Guidance, Control, and Dynamics, vol. 29(6), 2006, pp. 1295-1308

15. WERTZ, J. R, Spacecraft Attitude Determination and Control, Holland: Reidel Publication Company, 2002.

16. FORTESCUE, P., J. STARK, G. SWINERD, Spacecraft Systems Engineering, John Wiley \& Son, 2004.

17. SINGH, S. N., W. YIM, Nonlinear Adaptive Backstepping Design for Spacecraft Attitude Control Using Solar Radiation Pressure in the Proceedings of the 41st IEEE Conference on Decision and Control, Las Vegas, 2002, pp. 1239-1244.

18. V. J. LAPPAS, W. H. STEYN, C. I. UNDERWOOD, Attitude Control for Small Satellites using Control Moment Gyros, Acta Astronaut, 2002. 
19. DAVID, D. A., R. J. FUENTES, Adaptive Attitude Control and Closed-loop Power Tracking for an Integrated Power and Attitude Control System Using Variable Speed Control Moment Gyroscopes in the Proceedings of AIAA Guidance, Navigation, and Control Conference and Exhibit, San Francisco, 2005, pp. 1-23.

20. RICHIE, D., J. TSIOTRAS, P. FAUSZ, Simultaneous Attitude Control and Energy Storage using VSCMGs: Theory and Simulation, in the Proceedings of the American Control Conference, Arlington, 2001, pp. 3973-3979.
21. LAPPAS, V.,J., W. H. STEYN, C. I. UNDERWOOD, Practical Results on the Development of a Control Moment Gyro based Attitude Control System for Agile Small Satellites, in the Proceedings of the 16th Annual AIAA-PUSU Conference on Small Satellites, Logan, 2002, pp. 1-9. 